\title{
Strongly enhanced four wave mixing in micro-ring resonators integrated with 2D graphene oxide films
}

\author{
Jiayang Wu, ${ }^{1}$ Yunyi Yang, ${ }^{1}$ Yuning Zhang, ${ }^{1}$ Yang Qu, ${ }^{1}$ Linnan Jia, ${ }^{1}$ Xingyuan Xu, ${ }^{1}$ \\ Sai T. Chu, ${ }^{2}$ Brent E. Little, ${ }^{3}$ Roberto Morandotti, ${ }^{4,5}$ Baohua Jia, ${ }^{1}$ and David J. Moss ${ }^{1 *}$ \\ ${ }^{I}$ Centre for Micro-Photonics, Swinburne University of Technology, Hawthorn, Victoria 3122, Australia \\ ${ }^{2}$ Department of Physics, City University of Hong Kong, 83 Tat Chee Avenue, Hong Kong, China. \\ ${ }^{3} X i$ 'an Institute of Optics and Precision Mechanics Precision Mechanics, Chinese Academy of Science, Xi'an, China. \\ ${ }^{4}$ INRS-Énergie, Matériaux et Télécommunications, 1650 Boulevard Lionel-Boulet, Varennes, Québec, J3X 1S2, Canada.
}

\begin{abstract}
Two-dimensional layered graphene oxide films are integrated with micro-ring resonators to experimentally demonstrate enhanced four-wave mixing, achieving up to $~ 7.6-\mathrm{dB}$ enhancement in conversion efficiency for a uniformly coated device and $\sim 10.3-\mathrm{dB}$ for a patterned device.
\end{abstract}

\section{Introduction}

As a fundamental third-order $\left(\chi^{(3)}\right)$ nonlinear optical process [1], four-wave mixing (FWM has found wide applications in all-optical signal generation and processing, such as wavelength conversion, optical comb generation, quantum entanglement, optical sampling, and many others [2-5]. Integrated micro-ring resonators (MRRs), which confine light in compact micro-scale resonant cavities, are key building blocks for photonic integrated circuits [6-8]. Compared with FWM in waveguides, FWM in MRRs can provide dramatically enhanced conversion efficiencies (CEs) due to resonant enhancement of the optical field, thus significantly reducing the power requirements.

Although silicon has been a leading integrated platform for linear optics, for nonlinear optics it suffers from strong two-photon absorption (TPA) in the telecommunications band, which greatly limits the FWM performance [1]. Other integrated platforms such as silicon nitride and high index doped silica glass, though have a much weaker TPA, still face limitations in terms of FWM efficiency since their Kerr nonlinearity $\left(n_{2}\right)$ is over an order of magnitude smaller than that of silicon [9, 10]. Recently, [11] we demonstrated enhanced FWM in waveguides integrated with twodimensional (2D) layered graphene oxide (GO) films as well as high performance linear polarizers in waveguides and MRRs [12]. Here, [13] we report enhanced FWM in CMOS-compatible MRRs integrated with GO films. By using a large-area, transfer-free, layer-by-layer GO coating method together with photolithography and lift-off processes, we achieve precise control of the film thickness, placement, and coating length. Owing to the strong light-matter interaction in the MRRs integrated with highly nonlinear GO films, the FWM efficiency in the hybrid MRRs is significantly improved. We achieve up to 7.6-dB enhancement in the FWM CE for an MRR uniformly coated with 1 layer of GO and $\sim 10.3-\mathrm{dB}$ for a device patterned with 50 layers of GO. These results confirm the high nonlinear optical performance of integrated photonic resonators incorporated with layered GO films.

\section{Four-wave mixing in GO-coated MRRs}

Figure 1(a) shows microscopic images of an integrated MRR patterned with 50 layers of GO ( 50 $\mu \mathrm{m}$ pattern length). The MRR was fabricated on a high index doped silica glass platform using CMOS compatible fabrication processes $[11,12]$. Chemical mechanical polishing was used to remove the upper cladding, so as to enable GO film coating on the top surface of the MRR. The coating of layered GO films was achieved via a solution-based method that yields transfer-free [14], layer-by-layer GO film deposition [14]. Based on this GO coating technique, we achieved GO patterning on integrated photonic devices via photolithography and lift-off processes. Figure 1(b) shows a scanning electron microscope image of the 2D layered GO film, with up to 5 layers of GO (with a thickness of $\sim 2.25 \mathrm{~nm}$ on
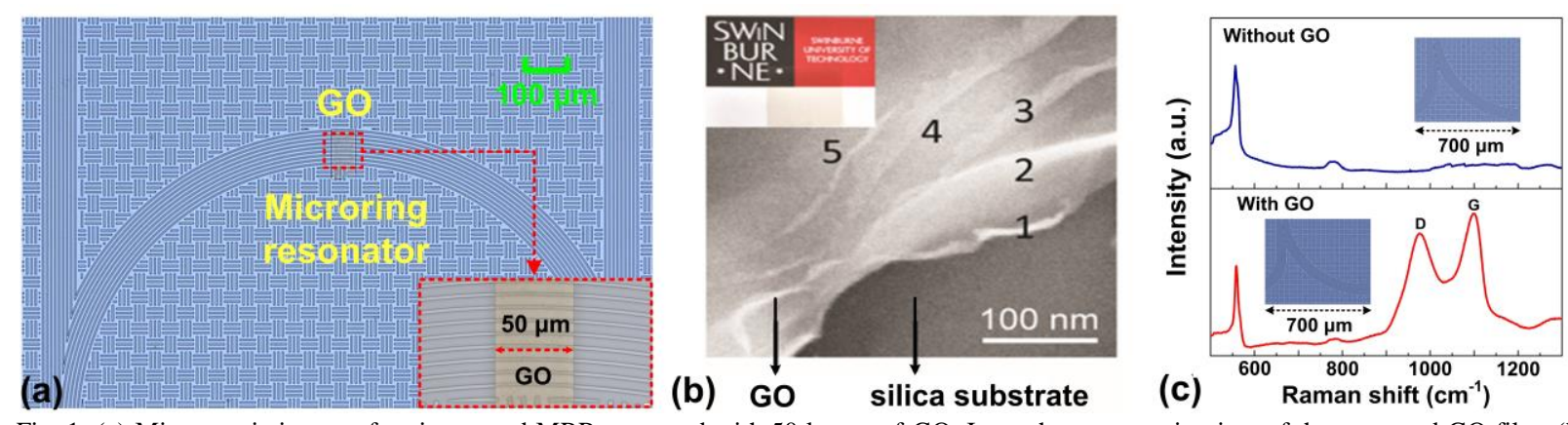

Fig. 1. (a) Microscopic image of an integrated MRR patterned with 50 layers of GO. Inset shows zoom-in view of the patterned GO film. (b) Scanning electron microscope image of 2D layered GO film. (c) Raman spectra of an integrated chip without GO and with 2 layers of GO. 
average for each layer). Figure 1(c) shows the measured Raman spectra. The presence of the representative D and G peaks of GO confirms the integration of GO film onto the top surface.
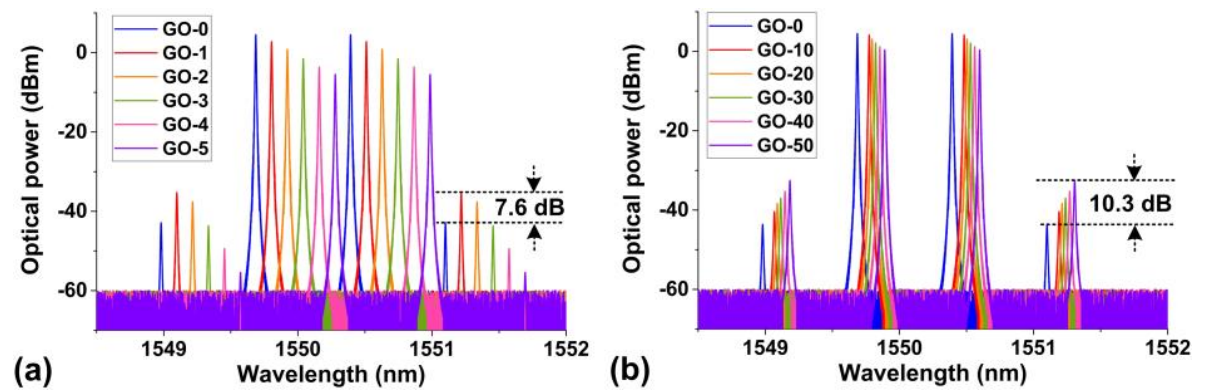

Fig. 2. Optical spectra of FWM at a pump power of $22 \mathrm{dBm}$ for the MRRs with (a) 1-5 layers of uniformly coated and (b) 10-50 layers of patterned GO films, respectively. The results for uncoated MRR (GO-0) are also shown for comparison.

Figure 2(a) shows the FWM spectra of the MRRs uniformly coated with 1-5 layers of GO, together with the FWM spectrum of the uncoated MRR. For comparison, we kept the same pump power of $\sim 22 \mathrm{dBm}$ coupled into the MRRs. As compared with the uncoated MRR, the GO-coated MRRs had an additional insertion loss, while the MRRs with 1 and 2 layers of GO clearly show enhanced idler output powers. The CE of the MRR without GO and with 1 layer of GO were $\sim-48.4 \mathrm{~dB}$ and $\sim-40.8 \mathrm{~dB}$, respectively, corresponding to a $\mathrm{CE}$ enhancement of $7.6 \mathrm{~dB}$ for the GO-coated MRR. Figure 2(b) shows the FWM spectra of the MRRs with 10-50 layers of patterned GO. The GO coating length was $\sim 50 \mu \mathrm{m}$ and the pump power $(22 \mathrm{dBm})$ was the same as that in Fig. 2(a). The results for all the tested GO layer numbers show enhanced idler output powers. In particular, there is a maximum $\mathrm{CE}$ enhancement of $\sim 10.3 \mathrm{~dB}$ for the MRR patterned with 50 layers of GO. Figures 3(a)-(c) show the FWM spectra versus $\Delta \lambda$ (wavelength spacing between pump and signal) for the uncoated MRR, the uniformly coated MRR with 1 layer of GO, and the patterned MRR with 50 layers of GO, respectively. The measured CE versus $\Delta \lambda$ is depicted in Figure 3(d) where we see that, for all three MRRs, the CE only shows a slight decrease with $\Delta \lambda$, reflecting the low dispersion of the doped silica MRR and the GO-coated MRRs, thus enabling effective phase matching for broadband FWM. Compared with other 2D materials, $n_{2}$ for GO is lower than graphene but still two orders of magnitude higher than bulk silicon [11-16], which demonstrates the high potential of GO as a new optical material for nonlinear photonic applications. Further, like Si-Ge heterostructures, $[17,18]$ GO may also offer interesting possibilities for both $2^{\text {nd }}$ and $3^{\text {rd }}$ order nonlinear effects courtesy of its complex anisotropic nonlinear optical characteristics.

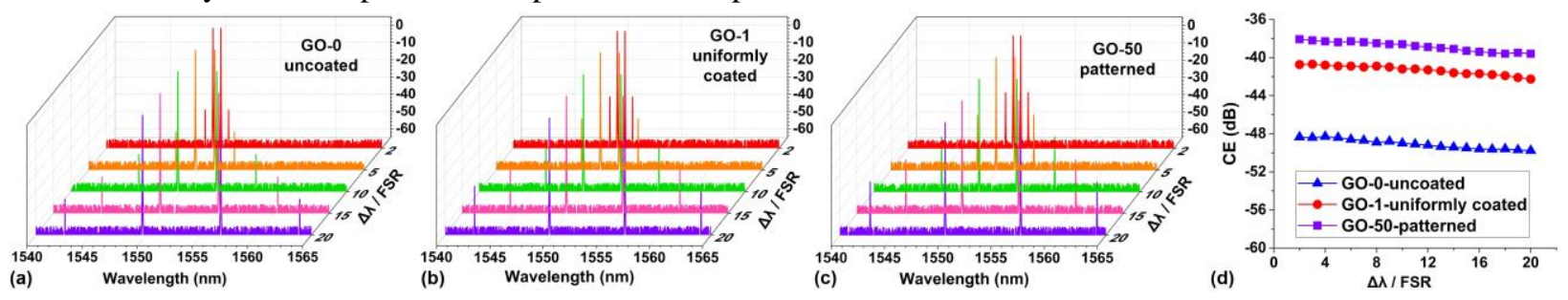

Fig. 3. (a)-(c) Optical spectra of FWM at different resonant wavelengths for the uncoated MRR, the MRR uniformly coated with 1 layer of GO, and the MRR patterned with 50 layers of GO, respectively. $\Delta \lambda$ and FSR represent the wavelength spacing between pump and signal and the free spectral ranges of the MRRs, respectively. (d) Measured CE versus $\Delta \lambda / F S R$ for the MRRs in (a)-(c). The pump power in (a) $-(d)$ was $22 \mathrm{dBm}$.

\section{Conclusion}

We demonstrate enhanced FWM in MRRs integrated with layered GO films. We perform FWM measurements for MRRs uniformly coated and patterned with GO films, achieving up to $~ 7.6-\mathrm{dB}$ and $\sim 10.3-\mathrm{dB}$ enhancement in the FWM CE for the MRRs uniformly coated with one layer of GO and patterned with 50 layers of GO, respectively.

\section{References}

[1] J. Leuthold, C. Koos, and W. Freude, "Nonlinear silicon photonics," Nat. Photon., 4(8), 535, 2010.

[3] M. Kues et.al., "On-chip generation of high-dimensional entangled quantum states and their coherent control," Nature, 546(7660), 622, 2017.

[4] A. Pasquazi et.al., "All-Optical wavelength conversion in an integrated ring resonator", Opt. Exp. 18(4) 3858, 2010.

[5] H. Bao , et.al, "Laser Cavity-Soliton Micro-Combs", Nature Photonics 13 (6) 384-389 (2019).

[6] J. Wu et.al., "Advanced photonic filters based on cascaded Sagnac loop reflector resonators in silicon-on-insulator nanowires" APL Photonics, 3(4), 046102, 2018.

[7] M. Xu et.al., "Push-pull optical nonreciprocal transmission in cascaded silicon microring resonators," Photon. J., 5(1), $2200307,2013$.

[8] J. Wu et.al., "Nested configuration of silicon microring resonator with multiple coupling regimes," Photon. Technol. Lett., 25 (6), $583,2013$.

[9] D. Moss et.al., "New CMOS-compatible platforms based on silicon nitride and Hydex for nonlinear optics," Nat. Photon. 7(8), $597,2013$.

[11] Y. Yang et.al., "Enhanced four-wave mixing in waveguides integrated with graphene oxide," APL Photonics, 3(12), 120803, 2018.

[12] J. Wu et.al., "Graphene oxide waveguide and micro-ring resonator polarizers," Laser Photonics Rev., 13(9), 1900056, 2019. 
[13] J.Wu et.al., "Enhanced nonlinear FWM in MRRs integrated with layered GO films", Small 16(16), 1906563 (2020).

[14] Y. Yang et.al., "Bottom-up fabrication of graphene on silicon/silica substrate via a facile soft-hard template approach," Sci. Rep., 5, 1, 2015

[15] Y. Zhang et.al, "Enhanced Kerr nonlinearity and nonlinear figure of merit in silicon nanowires integrated with 2D graphene oxide films", ACS Applied Materials and Interfaces 12 (29) 33094-33103 (2020).

[16] D.J. Moss, et.al, "Dispersion in the anisotropy for optical third harmonic generation in Si and Ge", Optics Letters 14 (1), 57-59 (1989).

[17] M.Sinobad et.al., "Mid-IR octave spanning supercontinuum generation to $8.5 \mu \mathrm{m}$ in Si-Ge waveguides", Optica $\mathbf{5}$ (4), 360-366 (2018).

[18] E. Ghahramani, D.J. Moss, and J.E. Sipe, "Second-harmonic generation in odd-period, strained, (Si)n(Ge)n/Si superlattices and at Si/Ge interfaces", Physical Review Letters, vol. 64, no. 23, pp.2815-2818 (1990). DOI:10.1103/PhysRevLett.64.2815. 